\title{
Tunable Compact UHF RFID Metal Tag Based on CPW Open Stub Feed PIFA Antenna
}

\author{
Lingfei Mo and Chunfang Qin \\ State Key Laboratory of Industrial Control Technology, Department of Control Science and Engineering, Zhejiang University, \\ Hangzhou 310027, China \\ Correspondence should be addressed to Lingfei Mo, lfmo@iipc.zju.edu.cn
}

Received 15 August 2011; Revised 9 December 2011; Accepted 28 December 2011

Academic Editor: Seong-Youp Suh

Copyright ( 92012 L. Mo and C. Qin. This is an open access article distributed under the Creative Commons Attribution License, which permits unrestricted use, distribution, and reproduction in any medium, provided the original work is properly cited.

For the ultrahigh frequency radio frequency identification (UHF RFID) metal tag, it always has the difficulties of compact designing, especially for the conjugate impedance matching, low antenna gain, and fabrication or environmental detuning. In this paper, a tunable compact UHF RFID metal tag is designed based on CPW open stub feed PIFA antenna. By changing the length of the open stub, the impedance of the PIFA antenna could be tuned in a large scale for conjugate impedance matching. The open stub makes it easy to tune the resonant frequency to alleviate the fabrication detuning or the environmental detuning, even after the manufacture. Moreover, the CPW structure of the open stub feed can resist the effects of the metallic surface and increase the antenna gain for the compact PIFA antenna. Modeling analysis and simulation are in good agreement with the measurement results. It showed that the UHF RFID metal tag could be designed compact with good performance based on the CPW open stub feed PIFA antenna.

\section{Introduction}

Ultra high frequency radio frequency identification (UHF RFID) is a long-range noncontact automatic identification technology being widely used around the world recently. A UHF RFID system is generally composed of a reader and a tag. The reader reads the information of the tag through radio frequency (RF) wave. The tag is composed of a chip and an antenna, with no internal battery. All the energy it needs is obtained from the RF wave transmitted by the reader [1]. Compared with the traditional bar code tag, the UHF RFID tag can be read and written over a long distance with a very high data rate, no matter whether the tag is soiled or dirty. So, UHF RFID technology has been adopted in the logistics supply, automatic manufacture, traffic management, property security, and so forth. In some applications, the UHF RFID tags need to be attached on the surface of metallic objects, such as steel plates and steel containers. However, when UHF RFID tags, especially those with dipolelike antennas, are placed on the metallic surface, the reading distance is reduced quickly, or even cannot be read. This is because the metallic surface boundary changes the radiation efficiency, impedance matching, resonant frequency, and radiation pattern $[2,3]$. For this reason, some special tags which can be applied to the metallic surface were designed, called the metal mountable tags or metal tags. Impedance matching is very important for the UHF RFID metal tag antenna design. As the input impedance of the UHF RFID tag chip is generally complex, the antenna impedance should be designed to be the conjugate impedance of the chip for impedance matching [1]. Therefore, the input impedance of the antenna should be adjusted flexibly to achieve good conjugate impedance matching. Microstrip antenna, patch antenna, and PIFA antenna, which can take the metallic surface as ground plane, are designed for RFID metal tags with different impedance matching methods [4-8].

Compact UHF RFID metal tag is widely used in practical applications. However, for compact antenna design, the impedance matching of these antennas is limited because of the limited antenna structure. The impedance of the compact antenna should be able to be tuned flexibly in a large adjusting scale to match different tag chips. Furthermore, 
for commercial UHF RFID tags manufacture, the impedance may not be matched well between the antenna and the chip because of the simulation error and the fabrication variability, such as the substrate permittivity difference, manufacturing difference, and chip impedance difference. To get good impedance matching and performance, some manufacturers use automatic laser or mill machine to adjust the antenna physical structure to tune the impedance of the antenna. For this purpose, the tag antenna should be designed to have an easy tuning structure, which is easy for laser milling machine to adjust and achieving good impedance matching. Impedance tuning is also very useful to alleviate the detuning effects due to the different metallic application environments [2]. Besides the impedance matching and tuning, antenna gain is another challenge for the compact RFID metal tag. Compact RFID metal tags always have lower antenna gain and shorter read range. PIFA antenna is widely used for the compact UHF RFID tag mountable on metallic objects [9-11]. However, with radiation patch size reduction, the antenna gain decreases and the impedance matching become difficult. Using two PIFAs can get better antenna gains [12, $13]$ and flexible impedance matching $[5,14]$. But the size would increase obviously. Therefore, all of these previous works cannot fit the requirement of compact profile, easy tuning, and satisfied antenna gain.

In this paper, a tunable compact UHF RFID metal tag antenna with a CPW open stub feed is proposed. Through PIFA antenna, the tag was compact designed. The impedance of the antenna could be tuned freely by changing the length of the open stub [15]. Because of the open stub design, the impedance matching can be tuned even after the fabrication of the tag. Together with the open stub, the CPW is used to resist the effects of the metallic objects [16] and improve the performance of antenna gain [17]. In Section 2, the considerations of the UHF RFID tag design are discussed first. Based on these considerations, the proposed antenna is described in Section 3, with antenna structure, theoretical modeling and simulation results. In Section 4, measurement results of a prototype based on this design are also provided. Finally, discussion and conclusions are presented in Section 5.

\section{UHF RFID Tag Design Considerations}

A typical passive RFID tag is composed of a chip and an antenna, with no internal battery. All the energy it needs is obtained from the electromagnetic wave transmitted by a RFID reader. In a passive back-scattered UHF RFID system, the reader transmits a modulated signal with periods of unmodulated carry wave, which is received by the antenna of the tag. When the chip of the tag is activated by the power from the antenna, it will send back its identification information by modulating the backscattered signal. The backscattered signal is modulated by switching the load impedance of the chip between two states [1]. Figure 1 illustrates the operation of back-scattered passive UHF RFID system.

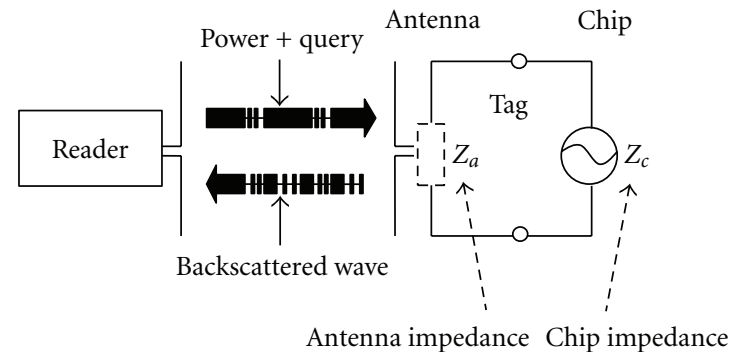

FIgURE 1: Principle of back-scattered UHF RFID system.

For UHF RFID tag, one of the most important criteria of performance is the read range. The maximum read range of the tag can be calculated as [18]

$$
r=\frac{\lambda}{4 \pi} \sqrt{\frac{P_{t} G_{t} G_{r} \tau}{P_{\text {th }}}}
$$

where $\lambda$ is the free space wavelength, $P_{t}$ is the power transmitted by the reader, $G_{t}$ is the gain of the antenna of the reader, $G_{r}$ is the gain of the antenna of the tag, $\tau$ is the power transmission coefficient between the tag antenna and the chip, and $P_{\text {th }}$ is the threshold power of the chip. When the reader and the chip of the tag keep the same, the maximum read range of the UHF RFID tag is mainly determined by the design of the tag antenna, especially the gain of the antenna of the tag $\left(G_{r}\right)$ and the power transmission coefficient $(\tau)$ [2]. The power transmission coefficient $\tau$ is determined by the impedance matching of the chip and the antenna, which can be calculated as follows:

$$
\tau=\frac{4 R_{c} R_{a}}{\left|Z_{c}+Z_{a}\right|^{2}}, \quad 0 \leq \tau \leq 1,
$$

where $Z_{c}=R_{c}+j X_{c}$ is the impedance of the tag chip, $Z_{a}=R_{a}+j X_{a}$ is the impedance of the tag antenna. When the impedances of the antenna and the chip are conjugate matching, the transmission coefficient $\tau$ could get the maximum value 1 and the most energy will be transmitted from the antenna to the chip when the tag is being enquired by the reader.

Besides the gain and the impedance matching, the bandwidth and the radiation pattern are also important considerations for UHF RFID tag antenna design. Wide bandwidth makes the tag to be read in a required bandwidth and the broadside radiation pattern makes the tag to be read in a wide direction scale $[19,20]$. In the realistic application, the size and shape of the tag must be designed to be embedded or attached to the target objects and have a reliable performance [21-23]. And for a commercial RFID tag, the cost is also an important requirement to be considered $[24,25]$. The cost of the RFID tag is a critical factor for this technology to be widely used around the world. Another requirement for RFID tag antenna design is the easiness for the mass production [26]. This includes the antenna manufacture, the chip bonding, the tag package, the performance testing, and the frequency tuning. The frequency tuning is useful for reducing the differences of chips and substrate materials, 
keeping the tags with the same performance before going to be used.

The requirements for designing a UHF RFID tag antenna are concluded in Table 1 . The proposed tunable compact tag antenna is designed according to these requirements.

\section{Proposed Antenna Design}

A tunable compact UHF RFID metal tag antenna is proposed in this paper. The PIFA antenna design makes the antenna compact than normal microstrip antenna. With an open stub feed, the antenna can be conjugate impedance matched with the chip easily by tuning the inset depth and the open stub length [15]. This metal tag antenna can be fabricated cheaply with normal PCB (Printed Circuit Board) technology. Moreover, with the open stub as tuning structure, the working frequency of the tag can be tuned even after the tag has been manufactured. The antenna bandwidth, radiation pattern, and metal stability also keep with good performance.

3.1. Antenna Structure. The structure and dimensions of the proposed antenna are illustrated in Figure 2. It is a planar inverted $F$ antenna with a shorting wall to reduce size. The radiation patch has dimensions of $W(20 \mathrm{~mm}) \times L(38 \mathrm{~mm})$ and is printed on a FR4 substrate $\left(\varepsilon_{r}=4.4, \tan \delta=0.02\right)$. The dimension of the substrate is $(W+2 \mathrm{~mm}) \times(L+$ $2 \mathrm{~mm}) \times h(3 \mathrm{~mm})$. The open stub feed line is inset into the patch to decrease the input impedance of the patch [27]. The inset structure has a length of $L_{\text {inset }}$ and a width of $W_{\text {inset }}$ $(8 \mathrm{~mm})$. The open stub feed line has a length of $L_{s}$ and a width of $W_{s}(3 \mathrm{~mm})$. The chip is attached on the feed port composed by the open stub line and the radiation patch. In order to enhance the gain of the compact PIFA antenna, a CPW structure is designed for the open stub feed line. The antenna is attached on a $200 \mathrm{~mm} \times 200 \mathrm{~mm}$ metal plate. The parameters $L_{\text {inset }}$ and $L_{s}$ are used as variables for impedance matching.

3.2. Theoretical Modeling Analysis. The transmission line model of the antenna is shown in Figure 3. From the antenna model, it is easy to know that the radiation patch and the CPW open stub feed lines are in series. Therefore, the input impedance of the feed port of the antenna can be calculated as

$$
Z_{\text {in }}=Z_{\text {in }}^{1}+Z_{\text {in }}^{2},
$$

where $Z_{\text {in }}^{1}$ is the input impedance of the radiation patch of the PIFA antenna, $Z_{\text {in }}^{2}$ is the input impedance of the CPW open stub feed line.

According to the basic RF circuit theory [28], the input impedance of the open stub can be simplified as

$$
Z_{\mathrm{in}}^{2}=-j Z_{0}^{2} \frac{1}{\tan \left(\beta L_{s}\right)}=-j Z_{0}^{2} \frac{1}{\tan \left(2 \pi L_{s} / \lambda\right)},
$$

where $Z_{0}^{2}$ is the characteristic impedance of the CPW open stub feed line, $\beta$ is the wave number, $L_{s}$ is the length of the CPW open stub feed line.
TABLE 1: Requirements for designing a UHF RFID tag antenna.

\begin{tabular}{lccc}
\hline $\begin{array}{l}\text { Requirements of UHF RFID } \\
\text { tag antenna design }\end{array}$ & \multicolumn{2}{c}{ Effects of improvement } \\
Read range & Cost & Reliability \\
\hline (1) Good impedance matching & $\sqrt{ }$ & & \\
(2) High antenna gain & $\sqrt{ }$ & & \\
(3) Wide bandwidth & $\sqrt{ }$ & & $\sqrt{ }$ \\
(4) Broadside radiation pattern & $\sqrt{ }$ & & $\sqrt{ }$ \\
(5) Compact size and shape & & $\sqrt{ }$ & \\
(6) Low manufacture cost & & $\sqrt{ }$ & \\
(7) Easy for testing and tuning & $\sqrt{ }$ & $\sqrt{ }$ & $\sqrt{ }$ \\
(8) Stable performance for use & $\sqrt{ }$ & & $\sqrt{ }$ \\
\hline
\end{tabular}

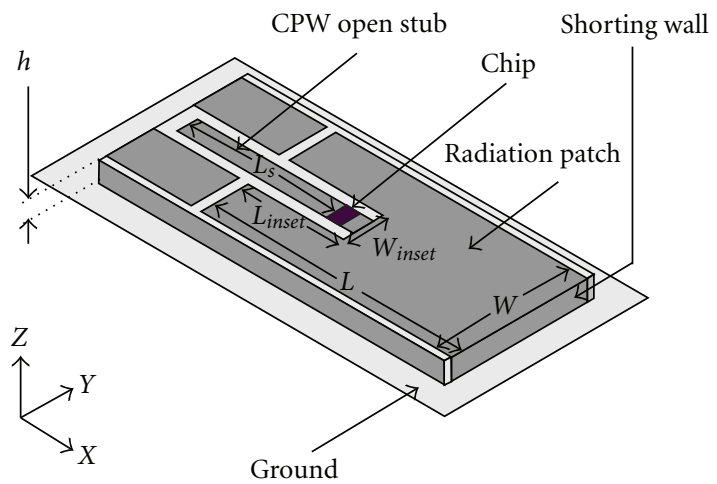

Figure 2: The open stub feed PIFA antenna structure. The dimensions of the radiation patch are $(L, W)$ while the open stub has dimensions $\left(L_{s}, W_{s}\right)$ and the height of the substrate (FR4) between the patch and the metallic surface is $h$.

The input impedance of the open stub only has imaginary part and its function of line length is shown in Figure 4. It shows that the reactance of the CPW open stub feed line is capacitive when the length is less than 0.25 wavelength and is inductive when the length is between the 0.25 wavelength and 0.5 wavelength. The reactance of the CPW open stub is a function of cotangent, which means that when the length of the open stub changes from 0 to $0.5 \lambda$, the imaginary part of the input impedance of the open stub changes from $-\infty$ to $+\infty$. Therefore, the imaginary part of the input impedance of the antenna can be tuned freely by the length of the open stub in a large scale.

3.3. Simulation and Optimization. In order to get a better impedance matching for the antenna, Finite-ElementMethod (FEM) based computational simulation software HFSS 12 is used for the simulation and optimization. For the UHF RFID tags, the chips generally have complex impedance, whose imaginary part is large and negative because of the rectifier and energy storage capacitor. In order to achieve the maximum energy transfer between the antenna and the chip, the input impedance of the antenna and the chip should be conjugate matching. That is, the real part is equal, and the imaginary part is opposite. As the imaginary part is much larger than the real part of the 


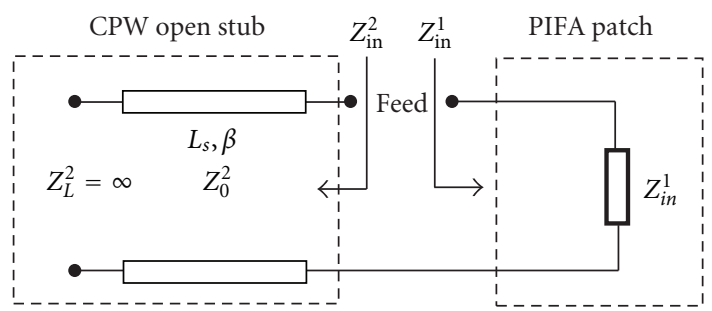

Figure 3: Transmission line model of the CPW open stub feed PIFA antenna.

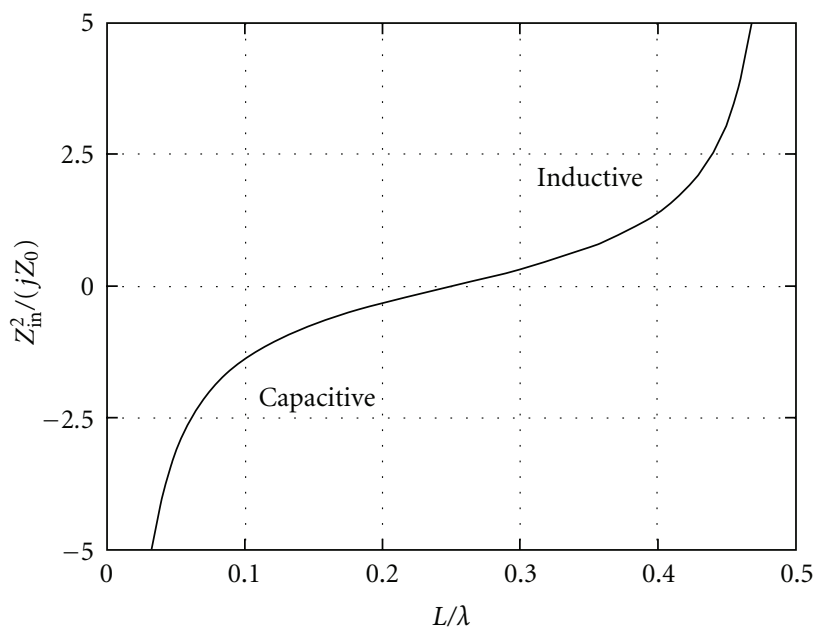

FIgURE 4: The input impedance of the CPW open stub feed line.

impedance, the impedance matching is mainly determined by the imaginary part matching. So, the antenna should be designed to have a structure easy for impedance tuning, especially for imaginary part tuning. As the proposed tag antenna in this paper is designed for the North America UHF RFID bandwidth (902 MHz 928 MHz), the tag antenna should have good impedance matching at this bandwidth. The chip used for the tag is the RI_UHF_00001_01 UHF RFID chip of TI (Texas Instruments), whose impedance is 9.9-j60.3 $\Omega$ at the frequency of $915 \mathrm{MHz}$. The structure of the antenna is shown in Figure 2. In order to simulate the tag antenna on the surface of metal, the tag is simulated on the surface of a reference metallic plate of $200 \times 200 \mathrm{~mm}^{2}$. According to the relative permittivity of the substrate, the length of the radiation patch $(L)$ of the PIFA antenna is chosen as $37 \mathrm{~mm}$, which makes the PIFA antenna resonant near the frequency of $915 \mathrm{MHz}$. The impedance matching between the antenna and the chip is tuned by $L_{\text {inset }}$ and $L_{s}$.

For patch antennas with the inset feed structure, increasing the depth of the inset could decrease the input impedance of the antenna [27]. Therefore, the length of the inset $L_{\text {inset }}$ can be used to tune the real part of the antenna impedance. Figure 5(a) shows the resistance tuning of the proposed antenna with different inset depths $\left(L_{\text {inset }}\right)$. The resistance of the antenna decreases with the increase of the inset depth $L_{\text {inset }}$. As we analyze above, the CPW open stub feed line can be used to tune the imaginary part of the input impedance of the antenna, which is shown in Figure 5(b). The reactance of the antenna increases with the increases of the CPW open stub length $L_{s}$. The imaginary part of the input impedance of the antenna could be tuned freely from $-\infty$ to $+\infty$ by changing the length of the CPW open stub from 0 to $0.5 \lambda$.

Therefore, for conjugate impedance matching of the proposal antenna, the resistance and the reactance could be tuned freely by the depth of the inset $\left(L_{\text {inset }}\right)$ and the length of the CPW open stub $\left(L_{s}\right)$, respectively. Through simulation and optimization, the parameters of the antenna are finalized as $L=37 \mathrm{~mm}, W=20 \mathrm{~mm}, h=3 \mathrm{~mm}$, $L_{\text {inset }}=14 \mathrm{~mm}$ and $L_{s}=30 \mathrm{~mm}$. With this dimension, the antenna input impedance and the reflection coefficient $S_{11}$ are calculated as shown in Figures 6(a) and 6(b), respectively. The imaginary parts of the impedance of the antenna and the chip are matched well at the frequency of $914 \mathrm{MHz}$. And the real parts of the impedance are matched at the frequency of $918 \mathrm{MHz}$. However, as the imaginary part of the impedance is much larger than the real part, the impedance matching is dominated by the imaginary part. Under this matching condition, the reflection coefficient $S_{11}$ is located at the $914 \mathrm{MHz}$ with a value of $-26 \mathrm{~dB}$. The $3 \mathrm{~dB}$ bandwidth of the antenna is $37 \mathrm{MHz}(895 \mathrm{MHz} 932 \mathrm{MHz})$, covering the bandwidth of the UHF RFID of North American. Moreover, with the decreases of the length of the open stub, the working frequency of the antenna is tuned from low to high.

\section{Measurement Results}

Based on the above-optimized parameters, the antenna sample was produced with an FR4 dielectric plate, as shown in Figure 7. The chip was attached to the antenna feed port with the traditional bonding technology. In order to test and compare the performance with the simulation results, the tag was also mounted on a $200 \times 200 \mathrm{~mm}^{2}$ copper plate. A commercial RFID reader, CSL-461 4-Port EPC Class1 Gen2 UHF RFID Reader [29], was used to test the tag. The bandwidth of the reader is $902 \mathrm{MHz} \sim 928 \mathrm{MHz}$. The output power of the reader can be tuned from $15 \mathrm{dBm}$ to $30 \mathrm{dBm}$, with a step of $0.25 \mathrm{dBm}$. The antenna of the reader is CS-771$2-\mathrm{R}$ with a gain of $6 \mathrm{dBi}$. Combining the output power of the reader and the reader antenna gain, the maximum radiation power is $36 \mathrm{dBm}(4 \mathrm{~W} E I R P)$. The reader and the tag are manufactured with the protocol of EPC Class1 Gen2 and ISO 18000-6C. According to the tag performance parameters and test methods of EPCglobal, the performance of the tag was measured based on the back-scattering method [30]. The maximum read range, power bandwidth, and radiation pattern were measured with the same method.

Through the back-scattering method, the best impedance matching frequency (resonant frequency) of the antenna could be measured. Because of the fabrication variability, the best impedance matching frequency is a little lower than $915 \mathrm{MHz}$. Then, as shown in Figures 5(b) and 6(b), through cutting the length of the open stub, the imaginary part of the impedance can be decreased and the resonant frequency can be increased to the target working frequency. In this way, the fabricated tag prototype is optimized by tuning the length 


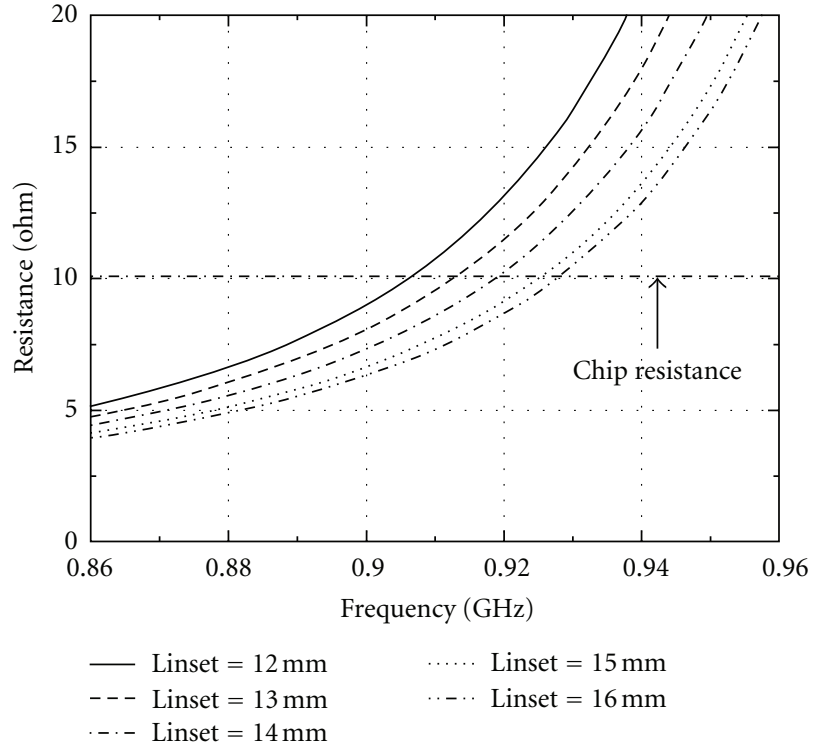

(a)

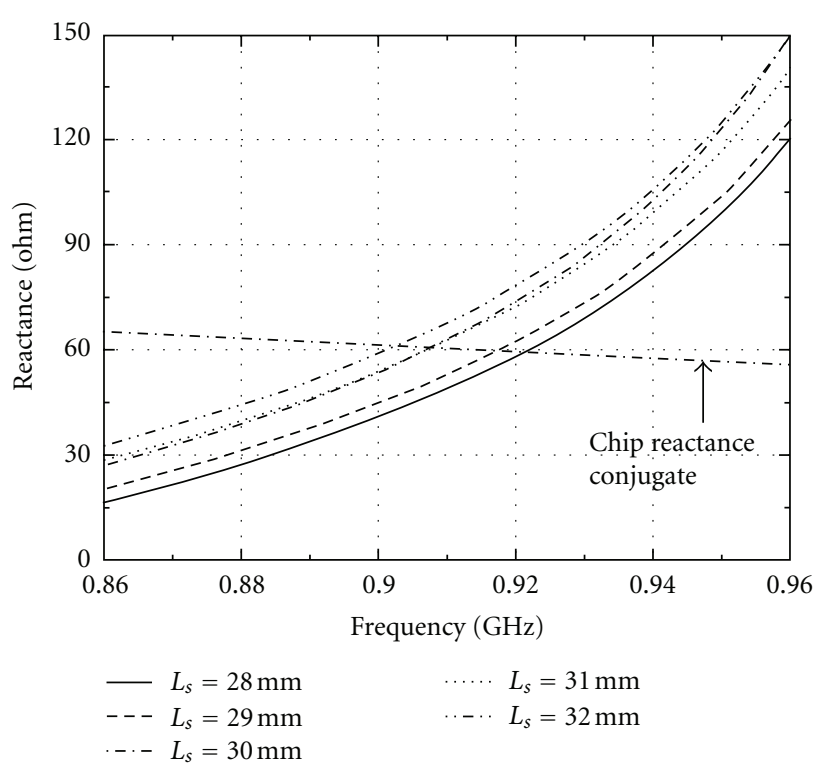

(b)

Figure 5: Antenna input impedance tuning. (a) Input resistance curves of the antenna with different inset depths $L_{\text {inset }}(L=37 \mathrm{~mm}, W=$ $\left.20 \mathrm{~mm}, h=3 \mathrm{~mm}, L_{s}=30 \mathrm{~mm}\right)$. (b) Input reactance curves of the antenna with different open stub length $L_{s}(L=37 \mathrm{~mm}, W=20 \mathrm{~mm}$, $\left.h=3 \mathrm{~mm}, L_{\text {inset }}=14 \mathrm{~mm}\right)$.

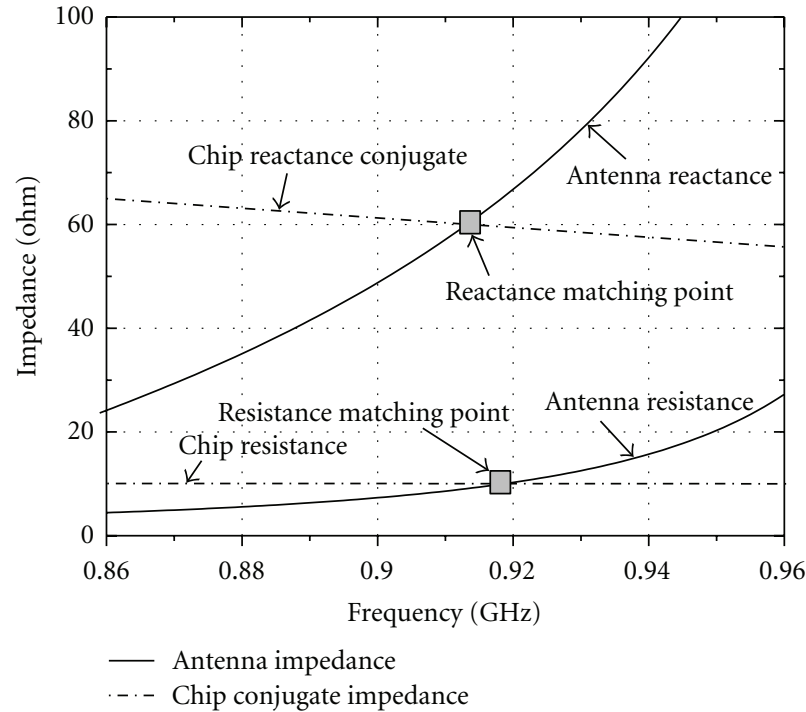

(a)

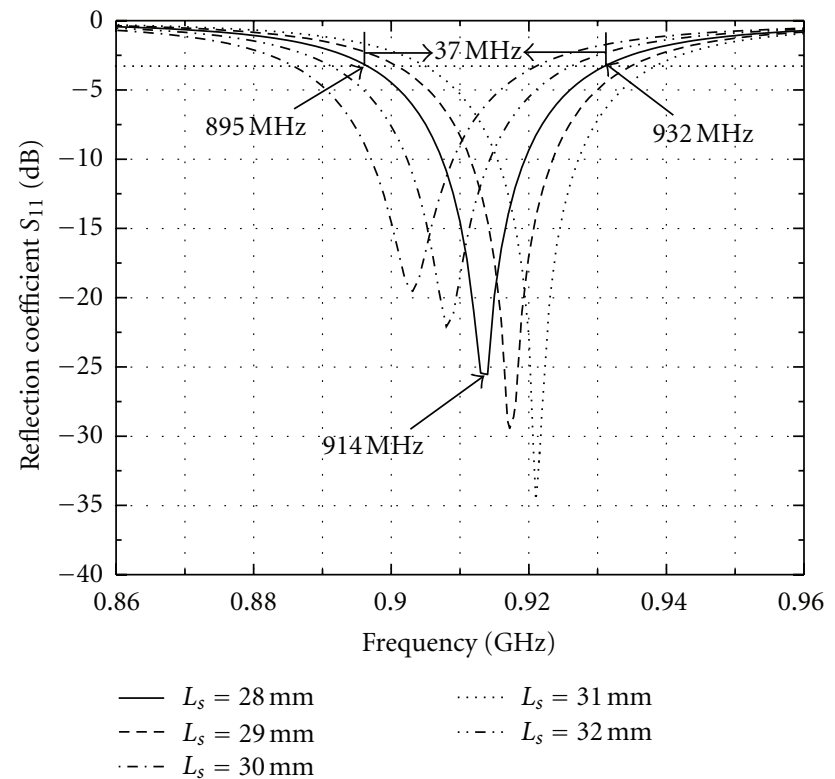

(b)

Figure 6: The input impedance and reflection coefficient $S_{11}$ of the antenna with optimized parameters: (a) impedance, (b) reflection coefficient $S_{11}$.

of the open stub to alleviate the detuning effects due to the fabrication process.

The maximum read range of the tag in North America bandwidth is plotted in the Figure 8. The antenna has a stable read range in the whole North America UHF RFID band with a max value of 4.7 meters at the frequency of $915 \mathrm{MHz}$. The tested results and the theoretical values are almost the same. The power bandwidth of the tag was measured in Figure 9. The output power of the reader needed to read the tags at different frequencies was normalized with the minimum value. The minimum is $0 \mathrm{~dB}$. From Figure 9, it is easy to calculate that the $3 \mathrm{~dB}$ power bandwidth is $903 \mathrm{MHz}$ $927 \mathrm{MHz}$, which covers most of the North America UHF RFID bandwidth. Compared with the $3 \mathrm{~dB}$ bandwidth of the 


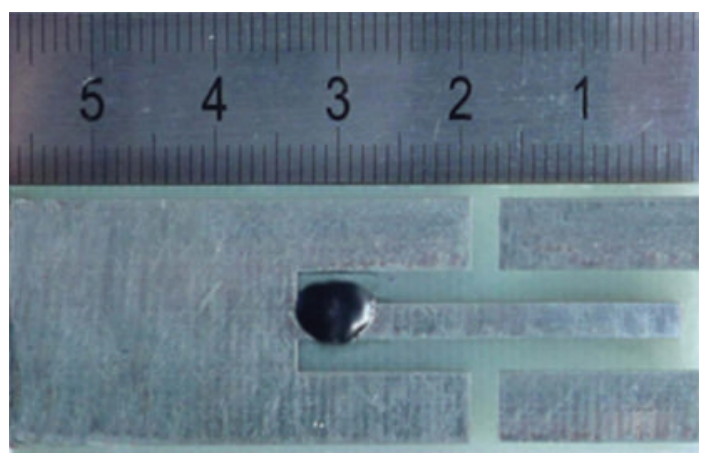

Figure 7: Photograph of the fabricated tag antenna.

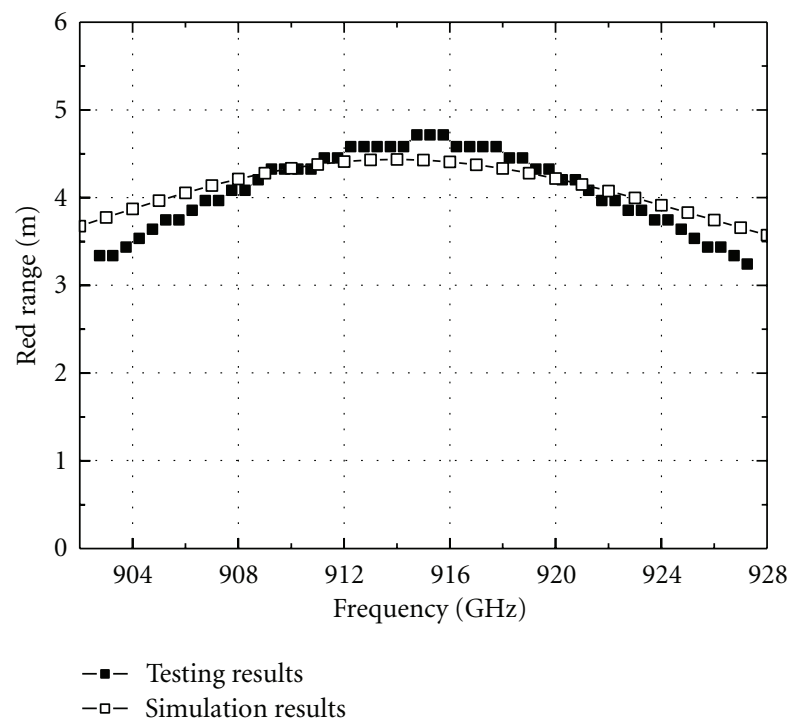

Figure 8: Theoretical and experimental read ranges for the open stub feed patch antenna $(E I R P=4 \mathrm{~W})$.

reflection coefficient $S_{11}$, the $3 \mathrm{~dB}$ power bandwidth is a little narrow, but the central bandwidth is almost the same. The measured radiation patterns of the tag at the frequency of $915 \mathrm{MHz}$ are shown in Figure 10. The antenna has nearly broadside hemisphere radiation pattern performance at both E plane and $\mathrm{H}$ plane. Tested results agree well with the simulation results. The tag was attached on the metallic plates of different size to test its metal performance. The testing results are plotted in Table 2, which shows that the metal tag has stable read range when it is placed on the surface of different metallic objects.

\section{Discussion and Conclusion}

A tunable compact UHF RFID metal tag based on CPW open stub feed PIFA antenna is designed in this paper. Using CPW open stub feed line, the impedance matching and antenna gain can be well designed. Moreover, because of the PIFA and the CPW structure, the antenna has stable performance for attaching on the surface of metallic objects. The working frequency of the antenna can be tuned by milling the length

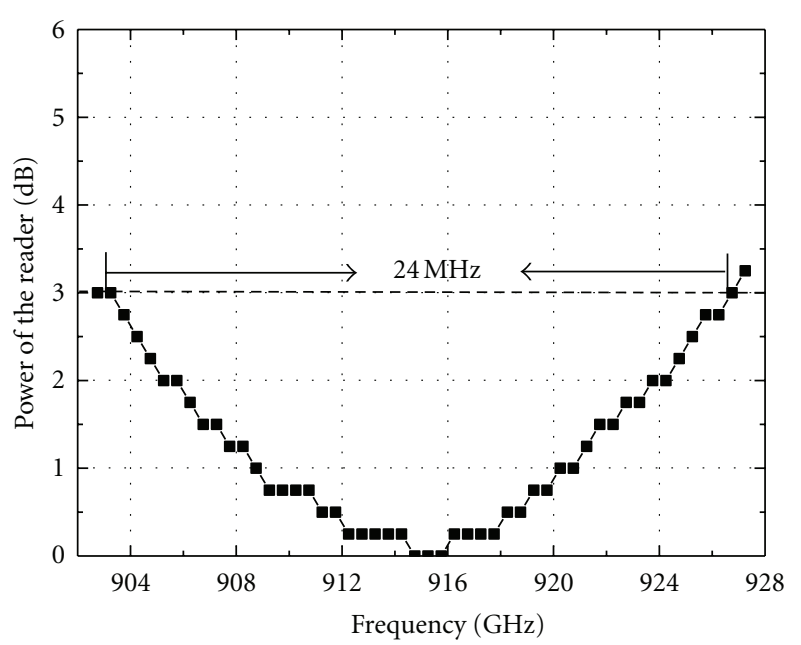

FIgURe 9: Power bandwidth of the tag antenna.

TABLE 2: Tag performance testing results with the tag attached on different metal plate.

\begin{tabular}{lc}
\hline Size of the metal plate & Read ranges \\
\hline $200 \mathrm{~mm} \times 200 \mathrm{~mm}$ & $4.7 \mathrm{~m}$ \\
$500 \mathrm{~mm} \times 500 \mathrm{~mm}$ & $4.8 \mathrm{~m}$ \\
$700 \mathrm{~mm} \times 700 \mathrm{~mm}$ & $4.3 \mathrm{~m}$ \\
\hline
\end{tabular}

of the open stub even after the tag has been fabricated. This can be used to alleviate the detuning effects of the fabrication error and the metallic application environments. With deceasing the length of the open stub, the imaginary part of the antenna can be reduced and the working frequency can be increased. With increasing the length of the open stub, the imaginary part of the antenna can be increased and the working frequency can be decreased.

The testing results were in good agreement with the simulation. This antenna has stable performance on different sizes of metallic objects. Four features can be concluded for this antenna design as follows.

(1) By PIFA antenna design, the size of the tag can be effectively reduced. The length of the PIFA antenna is only one half of that of microstrip antenna.

(2) An open stub feed is used to realize the impedance matching for this compact PIFA antenna. The impedance matching between the antenna and the chip could be achieved easily by tuning the length of the CPW open stub feed line. And this impedance matching method could be used with different chips and input impedances.

(3) With the CPW open stub feed line, the impedance matching of the tag could be tuned even after the manufacture of the tag. This makes it suitable for accurate impedance matching of the UHF RFID tag for manufacture and different application environments. 


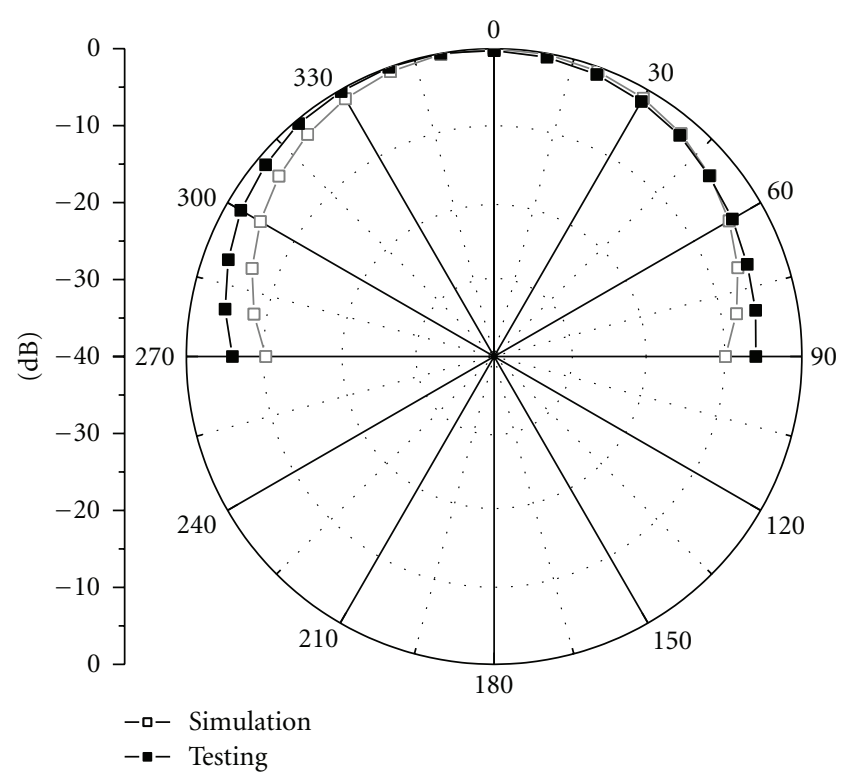

(a)

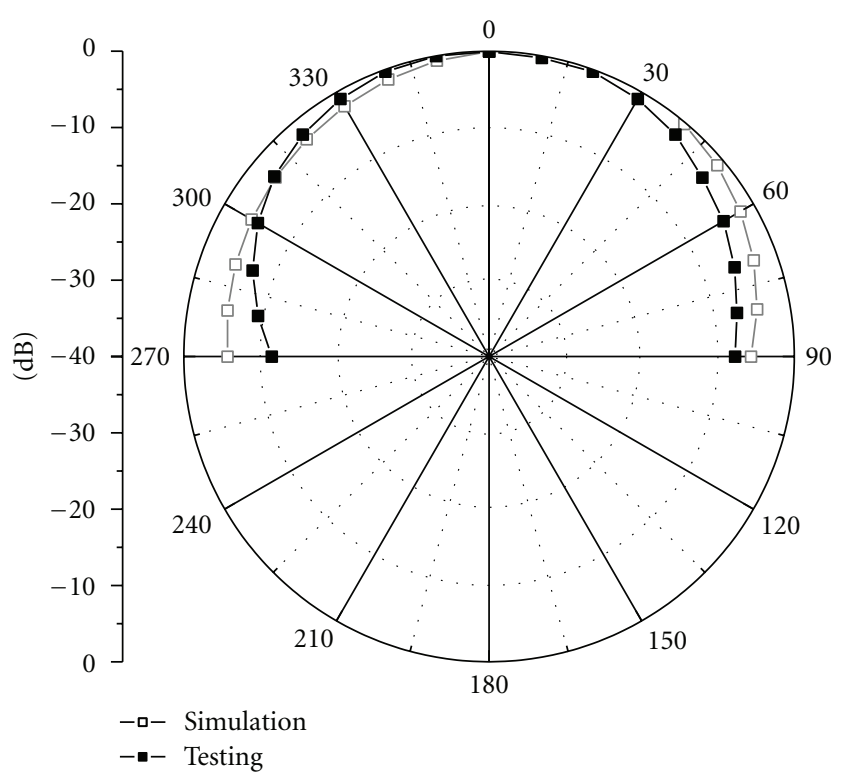

(b)

Figure 10: Radiation patterns of the open stub feed PIFA antenna: (a) E plane, (b) H plane.

(4) The CPW structure of the open stub feed can resist the effects of the metallic surface and increase the antenna gain for the PIFA antenna, which will keep the metal tag having a stable performance for attaching on the surface of different metallic objects.

\section{Acknowledgment}

This work was supported by the major projects of the Education Administration (Y200907699), Zhejiang province, China.

\section{References}

[1] K. Finkenzeller, RFID Handbook, John Wiley \& Sons, New York, NY, USA, 2nd edition, 2003.

[2] L. F. Mo, H. J. Zhang, and H. L. Zhou, "Analysis of dipolelike ultra high frequency RFID tags close to metallic surfaces," Journal of Zhejiang University A, vol. 10, no. 8, pp. 1217-1222, 2009.

[3] K. Penttilä, M. Keskilammi, L. Sydänheimo, and M. Kivikoski, "Radio frequency technology for automated manufacturing and logistics control. Part 2: RFID antenna utilisation in industrial applications," International Journal of Advanced Manufacturing Technology, vol. 31, no. 1-2, pp. 116-124, 2006.

[4] S. J. Kim, B. Yu, Y. S. Chung, F. J. Harackiewicz, and B. Lee, "Patch-type radio frequency identification tag antenna mountable on metallic platforms," Microwave and Optical Technology Letters, vol. 48, no. 12, pp. 2446-2448, 2006.

[5] B. Yu, S. J. Kim, B. Jung, F. J. Harackiewicz, and B. Lee, "RFID TAG antenna using two-shorted microstrip patches mountable on metallic objects," Microwave and Optical Technology Letters, vol. 49, no. 2, pp. 414-416, 2007.

[6] B. Lee and B. Yu, "Compact structure of UHF band RFID tag antenna mountable on metallic objects," Microwave and Optical Technology Letters, vol. 50, no. 1, pp. 232-234, 2008.
[7] K. H. Kim, J. G. Song, D. H. Kim, H. S. Hu, and J. H. Park, "Fork-shaped RFID tag antenna mountable on metallic surfaces," Electronics Letters, vol. 43, no. 25, pp. 1400-1402, 2007.

[8] H.-W. Son and S.-H. Jeong, "Wideband RFID tag antenna for metallic surfaces using proximity-coupled feed," IEEE Antennas and Wireless Propagation Letters, vol. 10, pp. 377380, 2011.

[9] M. Hirvonen, P. Pursula, K. Jaakkola, and K. Laukkanen, "Planar inverted-F antenna for radio frequency identification," Electronics Letters, vol. 40, no. 14, pp. 848-850, 2004.

[10] H. Kwon and B. Lee, "Compact slotted planar inverted-F RFID tag mountable on metallic objects," Electronics Letters, vol. 41, no. 24, pp. 1308-1310, 2005.

[11] W. Choi, H. W. Son, J. H. Bae, G. Y. Choi, C. S. Pyo, and J. S. Chae, "An RFID tag using a planar inverted-f antenna capable of being stuck to metallic objects," Electronics and Telecommunications Research Institute Journal, vol. 28, no. 2, pp. 216-218, 2006.

[12] J. S. Kim, W. Choi, and G. Y. Choi, "UHF RFID tag antenna using two PIFAs embedded in metallic objects," Electronics Letters, vol. 44, no. 20, pp. 1181-1182, 2008.

[13] S. L. Chen and K. H. Lin, "A slim RFID tag antenna design for metallic object applications," IEEE Antennas and Wireless Propagation Letters, vol. 7, Article ID 2009473, pp. 729-732, 2008.

[14] L. Xu, B. J. Hu, and J. Wang, "UHF RFID tag antenna with broadband characteristic," Electronics Letters, vol. 44, no. 2, pp. 79-81, 2008.

[15] L. Mo and C. Qin, "Planar UHF RFID tag antenna with open stub feed for metallic objects," IEEE Transactions on Antennas and Propagation, vol. 58, no. 9, Article ID 5484680, pp. 30373043, 2010.

[16] C. H. Ku, H. W. Liu, and P. J. Wang, "Novel CPW-fed slot antenna for UHF RFID metal tag applications," IEICE Electronics Express, vol. 8, pp. 410-415, 2011. 
[17] Y. Um, U. Kim, and J. Choi, "Design of a compact CPW-fed UHF RFID tag antenna for metallic objects," Microwave and Optical Technology Letters, vol. 50, no. 5, pp. 1439-1443, 2008.

[18] K. V. S. Rao, P. V. Nikitin, and S. F. Lam, "Antenna design for UHF RFID tags: a review and a practical application," IEEE Transactions on Antennas and Propagation, vol. 53, no. 12, pp. 3870-3876, 2005.

[19] L. Mo, H. Zhang, and H. Zhou, "Broadband UHF RFID tag antenna with a pair of U slots mountable on metallic objects," Electronics Letters, vol. 44, no. 20, pp. 1173-1174, 2008.

[20] M. Lai and R. Li, "Broadband UHF RFID tag antenna with parasitic patches for metallic objects," Microwave and Optical Technology Letters, vol. 53, no. 7, pp. 1467-1470, 2011.

[21] D. Kim and J. Yeo, "A passive RFID tag antenna installed in a recessed cavity in a metallic platform," IEEE Transactions on Antennas and Propagation, vol. 58, no. 12, Article ID 5582265, pp. 3814-3820, 2010.

[22] H. D. Chen and Y. H. Tsao, "Low-profile meandered patch antennas for RFID tags mountable on metallic objects," IEEE Antennas and Wireless Propagation Letters, vol. 9, Article ID 5419020, pp. 118-121, 2010.

[23] K. Ide, S. Ijiguchi, and T. Fukusako, "Gain enhancement of low-profile, electrically small capacitive feed antennas using stacked meander lines," International Journal of Antennas and Propagation, vol. 2010, Article ID 606717, 8 pages, 2010.

[24] S. Merilampi, L. Ukkonen, L. Sydanheimo, P. Ruuskanen, and M. Kivikoski, "Analysis of silver Ink bow-Tie RFID tag antennas printed on paper substrates," International Journal of Antennas and Propagation, vol. 2007, Article ID 90762, 9 pages, 2007.

[25] L. Yang, A. Rida, R. Vyas, and M. M. Tentzeris, "Novel "Enhanced-Cognition" RFID architectures on organic/paper low-cost substrates utilizing inkjet technologies," International Journal of Antennas and Propagation, vol. 2007, Article ID 68385, 7 pages, 2007.

[26] G. Orecchini, F. Alimenti, V. Palazzari, A. Rida, M. M. Tentzeris, and L. Roselli, "Design and fabrication of ultra-low cost radio frequency identification antennas and tags exploiting paper substrates and inkjet printing technology," IET Microwaves, Antennas and Propagation, vol. 5, no. 8, pp. 993 1001, 2011.

[27] R. Garg, P. Bhartia, L. Bahl, and A. Ittipiboon, Microstrip Antenna Design Handbook, Artech house publishers, London, UK, 2001.

[28] R. Ludwig and P. Bretchko, RF Circuit Design: Theory and Applications, Prentice-Hall, 2000.

[29] CSL. CSL-461 4-Port EPC Class1 Gen2 UHF RFID Reader, http://www.convergence.com.hk/.

[30] Tag Performance Parameters and Test Methods, EPCglobal Inc., 2008. 

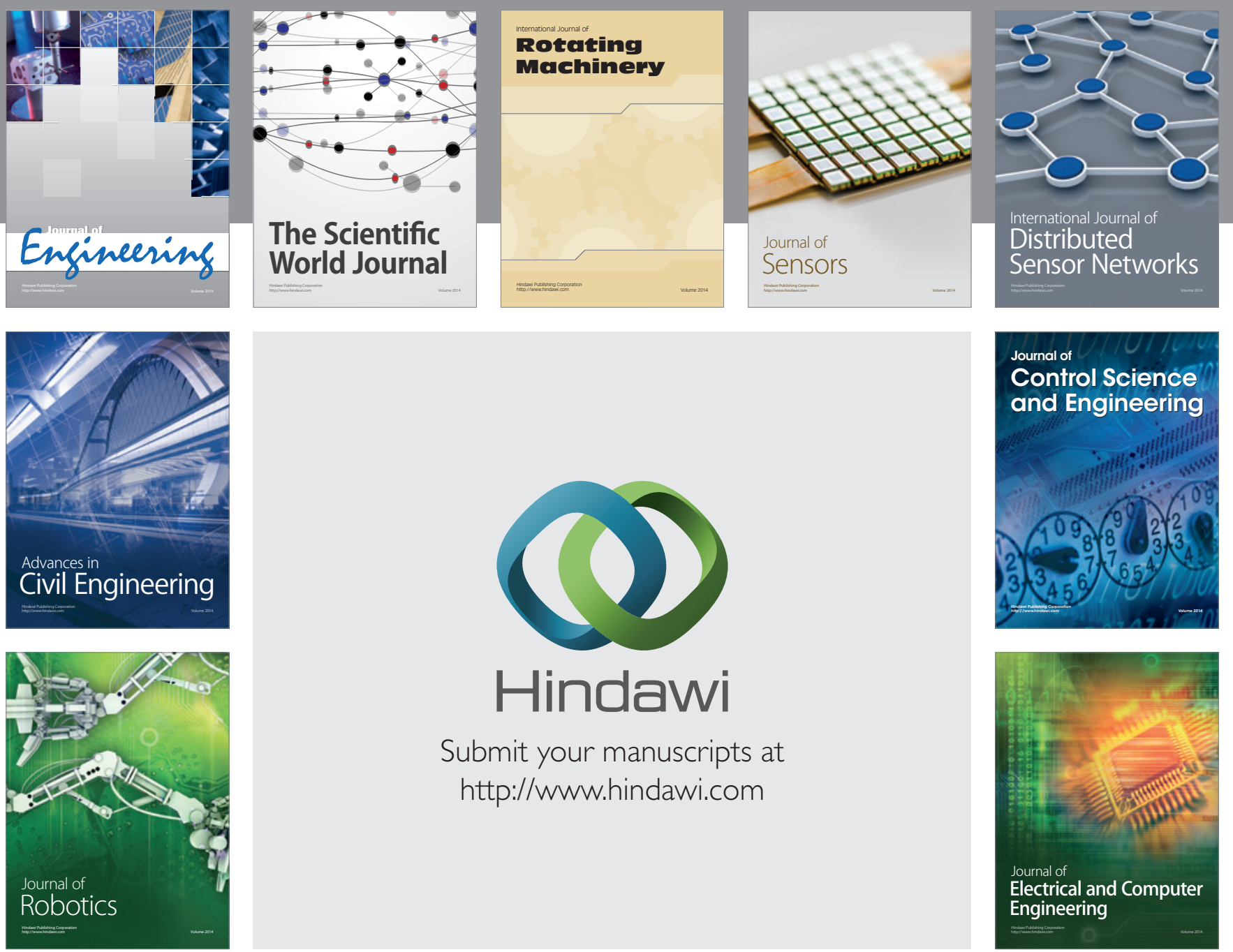

Submit your manuscripts at

http://www.hindawi.com
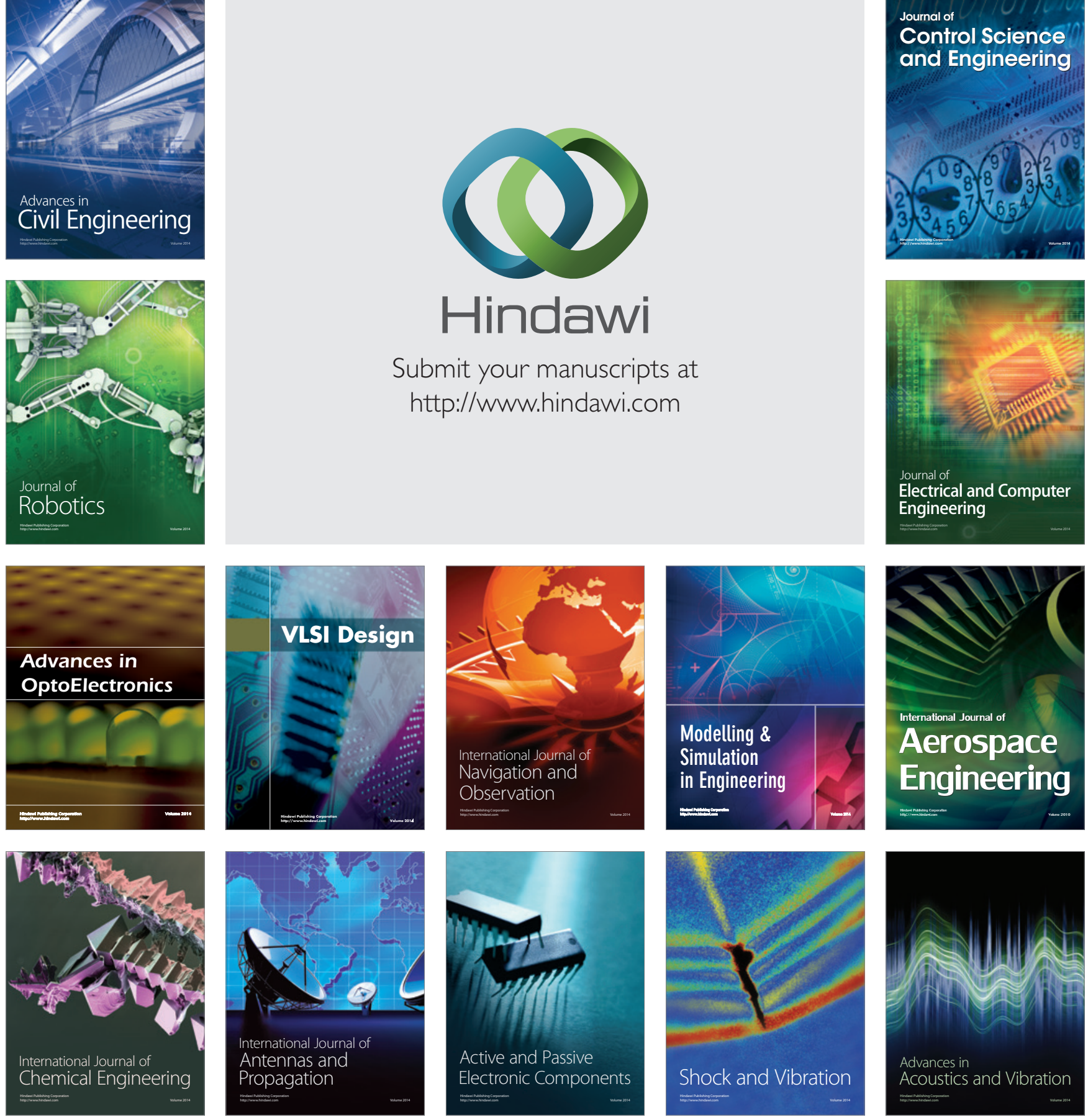